\title{
TTR
}

Traduction, terminologie, re?daction

\section{La migration des mots et le néerlandais comme « langue mineure » dans la mosaïque linguistique caribéenne}

\section{Kathleen Gyssels}

Volume 13, numéro 2, 2e semestre 2000

Les Antilles en traduction

The Caribbean in Translation

URI : https://id.erudit.org/iderudit/037416ar

DOI : https://doi.org/10.7202/037416ar

Aller au sommaire du numéro

Éditeur(s)

Association canadienne de traductologie

ISSN

0835-8443 (imprimé)

1708-2188 (numérique)

Découvrir la revue

Citer cet article

Gyssels, K. (2000). La migration des mots et le néerlandais comme « langue mineure » dans la mosaïque linguistique caribéenne. TTR, 13(2), 179-201. https://doi.org/10.7202/037416ar

\section{Résumé de l'article}

La migration des mots et le néerlandais comme « langue mineure » dans la mosaïque linguistique caribéenne - Parmi les diverses littératures antillaises, la littérature des îles néerlandaises est méconnue et peu étudiée. Cet article analysera d'abord les raisons de cette paucité et soulignera en même temps quelques rares initiatives visant à sortir de cet isolement linguistique : des numéros spéciaux de revues littéraires ont été dédiés à la promotion et à l'émancipation des auteurs caribéens-néerlandais. Dans un deuxième temps, l'article met l'emphase sur l'importance de la traduction en hollandais de la littérature antillaise francophone. L'éditeur hollandais Knipscheer a privilégié les auteurs caribéens francophones et nous nous penchons dans une dernière partie sur quelques-unes des difficultés que pose la traduction en hollandais des romans de Simone Schwarz-Bart et de Condé.
Tous droits réservés (C) TTR: traduction, terminologie, rédaction — Les auteurs, 2000 cotégé par la loi sur le droit d'auteur. L'utilisation des services d'Érudit (y compris la reproduction) est assujettie à sa politique d'utilisation que vous pouvez consulter en ligne.

https://apropos.erudit.org/fr/usagers/politique-dutilisation/ 


\section{La migration des mots et le néerlandais comme « langue mineure » dans la mosaïque linguistique caribéenne}

\section{Kathleen Gyssels}

Dans le dernier roman de Dominique Bona, le personnage-éditeur Jean Camus songe à la Caraïbe et aux différents auteurs qui peuplent cette région insulaire. Le manuscrit de Port-Ébène énumère les différentes îles et nomme leurs habitants :

La Guadeloupe et la Martinique, Saint-Martin figureraient alors à son catalogue, puis il y ferait entrer des traductions en anglais des îles Vierges, de Trinidad, de Sainte-Lucie et de La Barbade, enfin des auteurs flamands - l'autre côté de Saint-Martin, Curaçao ou l'île de la Marguerite -, [...]. (Bona, 1998, p. 53) (C'est moi qui souligne)

Ainsi Bona, "romancier antillais d'occasion", peuple-t-il la partie néerlandaise de l'île de Saint-Martin de Flamands, alors que ces derniers ne furent jamais colons dans cette région. De plus, le flamand n'est pas le néerlandais, mais plutôt une variante régionale de celui-ci. Ce passage illustre tout de suite la flagrante ignorance des auteurs français et de l'institution littéraire, relativement aux Antilles néerlandaises. Il donne un avant-goût de ce qui se passe dans le monde des lettres, tant dans le domaine de la formation du canon que dans la traduction.

\section{L'Écart (Mudimbé)}

Dans son introduction à The Other America: Caribbean Literature in a New World Context, le Barbadien Jean-Michael Dash souligne la 
nécessité, voire l'urgence, d'une perspective pancaribéenne, c'est-àdire d'une approche qui conceptualiserait la région comme un tout. Il s'excuse aussitôt de ne pas y inclure la Caraïbe néerlandophone dont, dit-il, si peu serait connu (Dash, 1998, p. XI). Alors même que Dash se propose d'explorer ce qui fait défaut, à savoir des analyses de l'héritage commun, des sensibilités parallèles qui rapprochent les littératures caribéennes au-delà des différences, son analyse reste incomplète, partielle, voire partiale. Dans un essai où Édouard Glissant et Antonio Benítez-Rojo côtoient Edward K. Brathwaite et Maryse Condé, aucune place donc pour les auteurs qui s'expriment en néerlandais. Non qu'ils soient inexistants, mais parce qu'ils restent inconnus. D'autres imputent cette lacune au fait qu'il s'agit d'u Îles miettes", comme le disait Césaire dans son Cahier d'un retour au pays natal. Ainsi, Jean-Marc Moura, tentant le mariage entre la théorie postcoloniale et les littératures francophones, explique dans une note en bas de page pourquoi les Antilles néerlandophones restent si méconnues: "Aux bases géographiques plus réduites, mais avec des auteurs antillais intéressants tels Tip Marrug (sic) ou Frank Martinus Arion » (Moura, 1999, p. 9). On ne voit pas pourquoi « les bases géographiques plus réduites » fourniraient la cause de l'ignorance des littératures en langue néerlandaise de la région. Sainte-Lucie, la Grenade, Antigue, les Antilles françaises ne sont pas plus grandes que Aruba, Bonaire ou Curaçao et cependant elles ont produit d' " énormes " auteurs, tels que le prix Nobel Derek Walcott, lequel revendique par ailleurs, dans The Schooner Flight, sa descendance hollandaise. Aimé Césaire et Édouard Glissant, George Lamming et V.S. Naipaul, pour ne nommer que ceuxlà, comptent parmi les plus grands de la littérature mondiale. Inacceptable comme excuse de cette négligence, l'exiguïté géographique est ce qui lie toutes les Antilles, les Petites comme les Grandes (Cuba, la Jamaïque et l'ancienne Hispaniola), l'insularité étant perçue par les auteurs comme un isolement, un emprisonnement. "I Îles cicatrices des eaux ", "Îles miettes ", "Îles informes", les vers de Césaire s'appliquent aux Antilles néerlandaises.

Dans La Isla que se repite (1989), le Cubain exilé Antonio Benítez-Rojo explore l'archipel caribéen dans son caractère plurilingue, mongrolisé et chaotique. Les Antilles néerlandaises n'y figurent pas. Même oblitération dans le manifeste littéraire des créolistes (Bernabé, Chamoiseau, Confiant) qui prétendent parler pour la région tout entière, et qui, sur la base d'une solidarité anthropologique, historique et linguistique, étendent la créolité aux communautés et littératures créolisées des Mascareignes, voire de l'Amérique (La Nouvelle- 
Orléans).

Il semble que Dash, Moura, Benítez-Rojo et les théoriciens martiniquais soient passés à côté d'un élément de taille, à savoir qu'il y a de fortes inégalités entre les différentes langues coloniales ellesmêmes, entre les langues européennes imposées aux peuples colonisés. La critique postcoloniale devrait y être sensible puisque le statut des littératures postcoloniales en dépend directement. Leur percée plus ou moins importante, bref, leur internationalisation et leur canonisation, sont « redevables » de la langue véhiculaire dans laquelle les auteurs ont choisi, voire ont été forcés, de par leur appartenance à l'une ou l'autre colonie, de s'exprimer. Si la littérature lusophone africaine, par exemple, est moins lue et étudiée que la littérature caribéenne de langue espagnole, c'est tout simplement parce que le portugais est moins important que l'espagnol. Il est clair que le succès des littératures afrocaribéennes de langue espagnole, française et anglaise est dû à la fois au fait que ces trois langues demeurent, avec le chinois, des langues mondiales. Elles bénéficient de la mondialisation, sont diffusées à l'échelle planétaire et sont l'objet de traductions. La troisième génération d'auteurs caribéens (la Dominicaine Loida Maritza Pérez, établie à New York, la Porto-Ricaine Lourdes Vázquez, Caryl Phillips, né à Saint-Kitts mais travaillant à New York et Londres) en profite en particulier. Marqués d'un exil volontaire, d'une identité rhizomatique (contre l'identité à racine unique, comme l'a définie Édouard Glissant dans sa Poétique $I V, 1997)$, ces nomades cosmopolites sont presque instantanément traduits. Edwidge Danticat, Haïtienne publiant en anglais à New York, Julia Alvarez, Dominicaine qui traite, comme Danticat, des relations tendues entre la République Dominicaine et Haïti, Ana Lydia Vega, Porto-Ricaine et Cristina Garcia ne sont que quelques-unes des nombreuses Caribéennes définies par au moins une double affiliation linguistique.

Dans Cuban-American Literature of Exile. From Person to Persona (1998), Isabel Alvarez-Borland nous convainc des insondables opportunités qu'offre le marché du livre américain, promoteur d'" ethnic literatures » et de «minority discourses ». Maryse Condé confirme ce constat en témoignant pour sa part des potentialités dont regorge le monde éditorial américain : pour une Antillaise d'expression française, la traduction en anglais accroît de manière exponentielle son lectorat. Sa résidence à New York, où elle est professeure de littérature à l'Université de Columbia, lui permet en même temps de noter d'importants changements dans le système littéraire. De fait, celui-ci 
est disloqué par le fait que le choix de la langue, à l'heure actuelle, devient de plus en plus, aux dires de la jeune génération migrante, une option stratégique, ou une contingence qu'il faut relativiser. Estimant que tout le débat autour de langue colonisatrice/colonisée est vain, chaque auteur étant colonisé par sa langue, elle est particulièrement sensible au fait que ceux qui sont originaires des îles caribéennes, et qui ont acquis ou achevé leur formation en Amérique du Nord ou en Europe, optent souvent pour une tierce langue, l'anglais (Danticat et Alvarez). D'autre part, Condé a été la première femme à recevoir le "Puterbaugh Prize for Fiction" (la plus haute distinction pour un auteur de langue étrangère aux É.-U.) pour l'ensemble de son cuvre romanesque, une distinction qu'elle n'aurait pas connue en restant chez elle en Guadeloupe.

Qu'il soit plus facile pour une femme noire de se faire publier, de trouver un lectorat aux Etats-Unis, et de se faire traduire, Condé l'illustre encore dans sa nouvelle "Trois femmes à Manhattan " (rééditée dans Pays mêlé, 1997). La Haïtienne Véra reste avec ses manuscrits sur les bras, alors que son amie africaine-américaine capte sans problème le regard des éditeurs et de la presse. En peu de temps, elle jouit d'une renommée extraordinaire. Les grandes villes, New York en tête, font le bonheur d'auteur(e)s et d'artistes de couleur. Dans une ère de migration toujours plus intense, effaçant d'anciennes frontières (géographiques, nationalistes, culturelles et linguistiques), c'est la traduction qui sert de levier à réaliser la globalisation littéraire, c'est-àdire à faire connaître ces littératures qu'on appela jusqu'à récemment " périphériques 》 ou " littératures des marges 》, ou encore, au Québec notamment, " littératures migrantes" : de belles collections comme " l'Étrangère » (Gallimard), " Domaine Étranger » (Bourgois), " Soul Fiction " (Éd. de l'Olivier), et " Haute Enfance » (Gallimard) jouent en même temps sur le besoin d'évasion des lecteurs modernes, satisfaisant leur triple dépaysement, dans l'espace, le temps, le Divers. Ce grand bouleversement révise le canon, plaide pour son élargissement. Comme l'a bien vu Carole Boyce-Davies dans Moving Beyond Boundaries (1995), une à une les frontières se renversent et les découvertes de nouvelles voix se font en respectant un ordre scrupuleux, déterminé par le prisme couleur, genre : après avoir sorti de l'ombre les auteurs caribéens, puis les femmes caribéennes, je dirais qu'il est temps de sortir du silence ceux qui ne publient pas dans une des trois langues européennes.

Dans ce collier d'îles où essaimèrent Espagnols, Anglais, 
Hollandais, Danois (et on peut s'étonner que ceux-ci n'aient pas laissé de trace littéraire), Français, la grande variété de langues officielles, doublée de celle des langues vernaculaires saute aux yeux. Cette abondance d'idiomes, cette véritable manne linguistique contraste singulièrement avec la fragmentation linguistique, les différentes communautés caribéennes s'ignorant jusqu'à tout récemment. La Dominique, aujourd'hui anglophone, entretient très peu de rapports culturels avec les îles voisines, la Guadeloupe et la Martinique, et vice versa. La barrière linguistique, séculaire, commence seulement aujourd'hui à s'effriter et les premières approches réellement comparatistes, incluant cette zone oubliée, à apparaître (voir les travaux de George Lang, 1997, 2000). Le phénomène de «balkanisation », né de la politique assimilationniste de la métropole, d'une part, le monolinguisme (chaque littérature insulaire se produisant dans la langue de l'ex-colonisateur), de l'autre, sont responsables de l'« écart " (pour employer le terme de V.Y. Mudimbé) qui sépara longtemps les différents champs littéraires caribéens. Les convergences ainsi que les divergences commencent à intéresser les critiques et les auteurs, stimulées aussi par les traductions bi-directionnelles (la collection " CARAF Books ", lancée par James A. Arnold, mérite d'être signalée à cet égard).

Le néerlandais est, du moins dans l'archipel caribéen, la langue la plus vulnérable dans le sens où elle a été imposée à une minorité de sujets coloniaux. À l'heure qu'il est, la littérature néerlandophone antillaise reste sous-représentée dans les anthologies et les " readers", dans les essais comparatifs comme dans les publications. Et ce, malgré quelques initiatives timides, dont des colloques et des revues. Quant à ces dernières, il me semble symbolique qu'elles soient américaines et/ou anglophones. Ainsi, Callaloo $(21.3,1998)$ présente le champ littéraire avec de larges extraits de traduction d'auteurs comme Helman, Roemer, Arion. Septentrion, revue française de culture néerlandaise, réserve régulièrement des articles à Arion. Ses poèmes dans Stemmen uit Afrika (1957), ses romans, Afscheid van de koningin (1975) et Nobele Wilden (19 y ont été étudiés avec des traductions de Jean-Philippe Riby (no 4, 1987, pp. 2-16, Gyssels, 1997). Signalons surtout l'initiative de la maison «In de Knipscheer, Major Publisher of Dutch Caribbean Literature at the Forefront of Multicultural Publishing ", qui lança une collection de traduction avec des noms comme Hugo Pos et Boeli Van Leeuwen. 
Je ne ferai que mentionner, ici, la richesse littéraire des Antilles néerlandaises et insister sur leur entrée, qui s'effectue lentement mais sûrement, dans la scène internationale littéraire, grâce à la traduction, non en français ou en espagnol, mais en anglais. Dans une seconde partie, je m'arrêterai à l'initiative inverse, qui consiste à traduire des auteurs des Antilles francophones en néerlandais, et je me pencherai plus spécifiquement sur quelques problèmes traductologiques avec Ton beau capitaine (Simone Schwarz-Bart 1987) et La Migration des cours (Maryse Condé 1996).

\section{La littérature afro-caribéenne néerlandophone}

Que suggère la liste fort incomplète des noms d'Edgar Cairo, de Thea Doelwijt, d'Ellis Juliana, de Tip Marugg, d'Anton de Kom, de Pierre Lauffer, de Hugo Pos, de Boeli Van Leeuwen, de Michaël Slory, de Trefossa, de Bea Vianen, de Ellen Ombre? Pour ceux qui connaissent les auteurs caribéens, chercheurs européens (français, anglais), canadiens, américains, cette série n'évoque pas grand-chose. Pourtant, le Surinam (l'une des trois Guyanes) et les Antilles néerlandaises partagent avec les autres littératures insulaires de la région la même donne littéraire, à savoir :

1. Diglossie : la langue officielle ou coloniale, le néerlandais, étant concurrencée par un grand nombre de langues indigènes au Surinam. Aux Antilles néerlandaises, différentes variantes du papiamentu coexistent, sans que l'inter-compréhension soit mise en danger : quelqu'un de Bonaire peut parfaitement comprendre quelqu'un de Curaçao, par exemple. Curaçao et Aruba peuvent se vanter d'avoir eu, dès le XIX ${ }^{\mathfrak{e}}$ siècle, une presse locale florissante en papiamentu. Dès les premiers "passants " (habitants blancs, allusion à leur résidence temporaire dans l'archipel), une littérature imprimée en papiamentu vit le jour dans les quotidiens et les magazines de l'époque (Broek 1990).

2. Métissage d'une tradition orale et d'une tradition livresque. Les Antilles néerlandaises ont une riche et longue tradition d'auratuur et d'oratuur, bien établie avant l'abolition, tardive dans ces colonies hollandaises, de l'esclavage (Rutgers 1994). Notons que cette date tardive, en comparaison avec les Antilles françaises (1848), pourrait être invoquée comme une raison historique du " retard " et donc, de la plus longue invisibilité, d'une production littéraire en néerlandais.

3. L'œuvre littéraire est pensée, mieux « imaginée » dans la langue 
maternelle, mais rendue, traduite dans la langue officielle; la langue dominante est soumise à une "indigénéisation ». Il se crée un " third code " (Chantal Zabus), une interlangue (Bill Ashcroft), les auteurs écrivant en présence permanente de deux langues. Plusieurs procédés (sémantiques, stylistiques, syntaxiques) réconcilient la langue-source et la langue-cible, les apports divers et multiples des idiomes " vernaculaires " (sonorité, sémantique, rythme, oraliture...) constituant une des dimensions les plus créatives de l'œuvre postcoloniale, qu'elle soit afro-caribéenne ou africaine. Toutefois, la tension constante entre les deux langues cause aussi la déculturation et l'aliénation, les " désastres" (" Hoquet », Léon-Gontran Damas, 1972, pp. 35-38). Le " code switching ", le changement de code linguistique en fonction de son interlocuteur et de la circonstance, ainsi que l'hypercorrection, sont symptômes d'insécurité linguistique et de surconscience, engendrées dans des contextes de diglossie et/ou de bilinguisme (Gauvin 2000). Il est question de "transliteration " (contamination de "translation 》 et "literation 》): " passeurs de langues », les écrivains des ex-colonies traduisent dans la langue paternelle leur sensibilité et leur imaginaire "créoles". La plupart des auteurs postcoloniaux perçoivent le bilinguisme comme une chance, un rempart contre « la fermeture identitaire ", contre "l'identité meurtrière " que redoutait par exemple Amin Maâlouf (Békri, 1999, p. 118).

4. À côté d'options esthétiques (et proprement stylistiques), il y a des options politico-idéologiques. Telle l'addition, en fin de volume, d'un glossaire, ou d'un appareil critique en bas de page. Ne pas gloser est un choix résolu de l'auteur postcolonial, accordant ainsi à la langue colonisée la position marquée.

5. La plupart des auteurs insulaires célèbres résident hors de l'île natale. La migration et la diaspora s'avèrent un avantage quand il s'agit de percer l'arène médiatique : Astrid Roemer, originaire du Surinam, vit depuis de longues années à La Haye et cela explique qu'elle soit la plus célèbre romancière antillo-néerlandophone. Een naam voor de liefde ( $A$ Name for Love, 1994) et De orde van de dag (The Order of the Day, 1994) ont paru en anglais.

6. Avec l'émancipation des langues indigènes, avec la « défense et illustration » du créole comme langue littéraire par les auteurs d'Éloge de la créolité, notamment, il se pose aussi, pour les Antilles néerlandaises, la question de savoir dans quelle mesure le papiamentu peut rivaliser avec le néerlandais? 
Je me limiterai, pour le domaine néerlandophone caribéen, à trois figures incontournables, et pour lesquelles la traduction, malheureusement, n'a fait que commencer. Les îles $A B C$ peuvent se vanter d'avoir, au moment où la négritude s'éveilla à Paris, en même temps que la Harlem Renaissance (Langston Hughes, Countee Cullen, Claude MacKay, sous l'égide de Césaire, Senghor et Damas), un Cola Debrot. Mijn zuster de negerin (Ma sour la négresse 1934/5) a pourtant été très rapidement traduit en anglais : My Sister the Negro (1958). Mais la valeur pionnière, la richesse de ce petit bijou reste sousestimée et sous-documentée. Diplomate, ambassadeur de la culture créole, réfléchissant dans ses essais sur une définition de la littérature néerlandophone antillaise, insistant sur les contacts avec les régions, Debrot évoque pour moi ce que fut Saint-John Perse pour les Antilles françaises : fin observateur de la vie coloniale dans l'archipel caribéen, analyste des rapports de race, de classe et de sexe. "My Sister the Negro » aborde l'amour interdit et tabou entre "l'homme blanc et la femme de couleur », thème bien caribéen, longuement analysé par Fanon dans son Peau noire, masques blancs.

Une autre figure de " nestor " attend une traduction française : Albert Helman (Paramaribo 1903-1996), auteur le plus prolifique du Surinam, voire le plus grand auteur pour toute la littérature des excolonies de la Hollande. De plus, Helman fut lui-même traducteur d'un slave narrative de la main d'une abolitionniste anglaise, Aphra Behn. Ses romans débutants (Zuid-Zuid-West 1926), ses nouvelles (" Mijn aap schreit » 1928), et sa chronique des cinq Guyanes (De foltering van Eldorado. Een ecologische geschiedenis van de vijf Guyana's 1983) lui valurent le surnom de Multatulli du Surinam. De fait, son monument mémorial sur les Guyanes évoque celui d'Eduardo Galeano dans Mémoire du feu (1992). Son œuvre embrasse plusieurs domaines (la musique, l'ethnologie, la sociologie, l'histoire) et englobe tous les genres (poésie, nouvelles, romans, critique, théâtre, traductions, essais). Il est pour le Surinam ce qu'Alejo Carpentier et Cortázar sont respectivement pour les littératures cubaine et argentine. Surnommé le Naipaul du Surinam, ce grand érudit reste inconnu hors des Pays-Bas et de son pays natal. C'est qu'il manque des traductions (exception faite de Chieftains of the Oayapok!, Knipscheer, 1983) de cet « Indien retors et récalcitrant ».

La troisième figure échappe au cloisonnement linguistique, profitant des nouveaux outils de communication qui accélèrent la diffusion des œuvres d'un auteur, en même temps qu'ils bâtissent des 
ponts avec d'autres zones linguistiques. Frank Martinus Arion (Curaçao 1936) illustre à lui seul la créolité, version néerlandaise. Adepte de la «poétique de la relation " préconisée par Édouard Glissant, Arion fonda sa propre école en papiamentu, convaincu de la nécessité de plaider pour la reconnaissance de cette langue maternelle. Peu à peu, son approche translinguistique et sa perspective comparatiste fondée sur le partage d'une même histoire de subordination trouvent écho dans les milieux académiques. Héraut de la culture et de la littérature (et de l'éducation) en papiamentu, Arion publia en néerlandais des romans psychologiques, nous entraînant dans les profondeurs sinueuses des rapports homme-femme dans la société contemporaine de Curaçao, où bien des séquelles esclavagistes peuvent être observées. Son premier roman, Dubbelspel (1972), sortit en traduction dans la collection dirigée par Caryl Phillips (incluant le Guyanais Wilson Harris et Maryse Condé). Double Play (1998) signifie l'accès à un vaste public anglophone, et la différence se mesure aux maints débats, salons et conférences où il est depuis invité. En d'autres termes, la traduction consacre un auteur qui pourtant publie depuis les années 70 .

\section{La Migration des mots dans Ton beau capitaine}

Dès les années 70, la maison In de Knipscheer à Amsterdam saisit l'importance d'une auteure comme Condé. Son œuvre entière (ainsi que ses essais Le roman antillais et La poésie antillaise, parus en 1976 et 1977 chez Nathan) y ont été traduits. In de Knipscheer publia également une édition bilingue du Cahier d'un retour au pays natal (Aimé Césaire) et fit traduire tous les romans de Simone Schwarz-Bart. En 1991, Franc Knipscheer offrit en guise de cadeau amical à ses amis, clients et traducteurs la traduction de Ton beau capitaine (1987), pièce de thêâtre écrite à l'occasion des Troisième rencontres théâtrales caribéennes. Jouée par un seul acteur, cette pièce en un seul acte représente le drame d'un couple en même temps que l'incompréhension entre deux communautés antillaises francophones. Wilnor, ouvrier saisonnier haïtien venu couper la canne en Guadeloupe, bute en Guadeloupe contre l'animosité et la froideur; il y est considéré comme " le moins que rien ", le " nègre de nègres ». Il correspond avec MarieAnge, sa femme, par voie de cassettes. Après trois mois d'attente, Wilnor apprend que Marie-Ange l'a trompé avec le messager qui lui transmet l'argent durement gagné. Pire, qu'elle est enceinte de lui. Wilnor enrage, puis se calme à l'écoute de la voix de sa " femmecassettes ", de la musique envoûtante, après avoir dansé et imploré les dieux/orissa ou " loas » dans une espèce de transe. 
Schwarz-Bart retourne à deux sujets de prédilection : parler pour ceux et celles qui n'ont point de bouche, les « nègres des nègres », les Haitiens traités comme des subalternes (Spivak) en Guadeloupe. D'autre part, elle créolise le français (Bernabé 1979). « À la croisée de deux langues » (Gauvin 1997), Schwarz-Bart publie un texte parfaitement intelligible pour tout francophone. S'y tissent cependant des mots qui résonnent particulièrement pour un créolophone, tels que " malpropre » (putain), " plancher» (pour " lit» dans la formule : accepter un homme sur mon plancher), " rétréci du dedans et rétréci du dehors " (pour amaigri mais aussi moralement épuisé, las).

Dans ce texte éminemment court et sobre, il se produit un " marronnage linguistique ", une résistance à la dominance française. Comme le rappelle Glissant dans Le Discours antillais (1981, pp. 238239) : "le non-sens charroie le sens véritable ". "Pour l'Antillais, le mot est d'abord son "; contes, proverbes, devinettes attestent certes de l'oralité, mais relèvent aussi d'une pratique de camouflage et de détournement du sens. Ainsi, le "détour" se manifeste par l'usage d'images concrètes à sens abstrait. Par exemple, Wilnor a fort maigri, mais Marie-Ange emploie le mot "rétréci» pour désigner sa zombification. D'autre part, la juxtaposition de deux monologues met en relief une poétique du silence, traduisant avec succès l'exil et la souffrance, la solitude et le désespoir pour ceux qui sont marginalisés et victimes de discrimination.

De plus, certains syntagmes et expressions jaillissent directement de l'imaginaire schwarz-bartien. Ton beau capitaine regorge d'autotextualité. De multiples échos aux romans [rêves, proverbes ("l'homme est un rat, la femme est une rate »), oraliture, quadrille, lagghia, léroz, etc...] attestent de la cohésion de l'écriture schwarz-bartienne, jusque dans ses facettes linguistiques. Prenons un mot comme "clique », employé par Marie-Ange lorsqu'elle salue son homme, en même temps qu'adressant le bonjour et le bonsoir à tous les exilés caribéens, où qu'ils soient et d'où qu'ils viennent :

Bonnes nouvelles de tous nos exilés de par le monde: Grenade, Saint-Domingue, Portorique et la clique, ils vont bien et t'adressent le bonjour. (Schwarz-Bart, 1987, p.14)

Dans l'incipit de Ti Jean L'horizon, le mot renvoya à des déplacés, un groupe d'égarés (dont il est question dans Pluie et vent sur Télumée Miracle et qui fondent le morne La Folie), bref, des insulaires qui sont 
rongés par l'idée de ne pas être chez eux :

Et [la Guadeloupe] nourrit toutes qualités d'êtres étranges, hommes et bêtes, démons, zombis et toute la clique, à la recherche de quelque chose qui n'est pas venu, et qu'ils espèrent vaguement, sans en savoir la forme ni le nom; elle sert aussi de halte aux oiseaux qui descendent pondre leurs œufs au soleil... (Schwarz-Bart, 1979, p. 9)

Peuplée de « créatures de sable et de vent », « d'êtres étranges, hommes et bêtes, démons, zombis et toute la clique " (Schwarz-Bart, 1979, p. 9), la Guadeloupe serait donc une île à la dérive dont le peuplement hétéroclite est malheureux. Les mots français rayonnent d'une connotation créole (" qualité/toute la clique ») auto-dépréciative : dans la bouche du narrateur, qui feint ici l'observateur eurocentriste, incapable d'intégrer l'Autre, la Guadeloupe est une terre qui s'effrite, qui s'en va à vau-l'eau et où errent zombis et lunatiques. "Clique » renvoie au nombre infini, à la grande quantité d'immigrés et de déracinés de par le monde. Dans la traduction néerlandaise, " portorique et la clique » perd cette connotation autodépréciative, cette ironie aussi à l'égard de son propre sort migratoire.

Un autre problème est la traduction de termes français à connotation créole; bien qu'ils parlent français, les deux personnages tissent leur propre langage. Tout en empruntant la langue du colonisateur, Wilnor et Marie-Ange encodent leur propre langage, exprimant leur altérité. Schwarz-Bart veut celle-ci irréductible, intraduisible. D'où une certaine opacité, une épaisseur linguistique (Degras, 1995, p. 87), d'autant plus paradoxale que, contrairement à l'hermétisme d'un Glissant, Schwarz-Bart opte toujours pour un langage apparemment clair, peu docte. Cette langue simplissime se tisse néanmoins d'opacités. Les deux monologues de Wilnor et de Marie-Ange sont tout, sauf savants, langage de paysans. De surcroît, le silence prend ici une place dominante; le non-dit diffère le sens; le discours est cousu de silences. Schwarz-Bart répare l'oblitération de ces personnes/personnages qui se sont tus, et de ce fait, qui sont restés en marge, inécoutés, invisibles, exactement comme les histoires tribales des Native American (Vizenor 1994, pp. 142-143).

Dans toute traduction interculturelle, le traducteur a affaire à un autre univers qu'il doit par conséquent tenter de s'approprier; il est important qu'il connaisse le contexte de l'histoire du texte-source. Certains référents se déconnectent de leur ancrage socio-culturel en "transfusant» dans l'autre langue. Prenons l'exemple d' écume". 
Marie-Ange relativise son aveu de tromperie (le fait qu'elle ait dormi avec le messager). Elle s'explique sur son silence en disant que " l'histoire qui lui est restée dans la gorge est en fait rigolote ", qu'elle ne vaut pas " tant d'écume pour si peu de chocolat 》. Le mot " écume » est attesté plusieurs fois dans Pluie et vent sur Télumée Miracle :

1. le «négre » est "un poisson écumant, un poisson maigre à la renverse dans une assiette, un crabe sans tête, sans pinces, un chien sans pattes » (Schwarz-Bart, 1972, p. 154, pp. 158-159, p. 164).

2. "Mes yeux étaient deux miroirs dépolis et qui ne reflétaient plus rien. Mais lorsqu'on m'amena des vaches écumantes, le garrot gonflé de croûtes noires, je fis les gestes que m'avait enseignés man Cia et l'une d'abord, puis l'autre, les bêtes reprirent goût à la vie " (SchwarzBart, 1972, p. 226).

3. D'Élie, Reine sans Nom dit sagement à Télumée qu'« Il y a le bouillon et l'écume du bouillon et voilà ce qui en est de l'homme, écume et bouillon à la fois... mais ce n'est que l'écume du bouillon qui poursuit Élie, rien que l'écume et elle ne tarira pas demain, non... c'est pourquoi je te le dis, si tu ne fuis pas pendant qu'il en est encore temps, elle te submergera » (Schwarz-Bart, 1972, p. 156).

Substance mousseuse sur le museau de poissons et de vaches agonisantes, substance graisseuse qu'on écope du bouillon, l'écume a un troisième référent : substance qu'on obtient après cuisson du jus de canne, l'écume surconnote l'univers de la plantation, de la canne à sucre et de la fabrication de rhum. Contrairement aux mélasses, qui sont recyclées, l'écume constitue le résidu non recyclable. Résidu sans qualité, il est éliminé dans la distillation d'un rhum de bonne qualité (et non "l'eau de mort guildive». Traduire «tant de vent pour une si petite barque, tant de mousse pour si peu de chocolat » par « zo veel sop voor zo'n klein kooltje » (littéralement : " tant d'eau pour un si petit chou» au sens de "le jeu n'en vaut pas la chandelle») fait abstraction du référent à " l'univers de Plantation " (pour parler avec Glissant). Chamoiseau et Confiant avaient déjà attiré l'attention sur ce langage " insondable » dans le romanesque schwarz-bartien, au point de l'appeler le "téluméen " (Chamoiseau \& Confiant 1991, p. 181). "Écume» est un des multiples sèmes sur lesquels viennent se sédimenter, se surimposer, plusieurs référents : si le Martiniquais ou le Guadeloupéen avoue la complexité du langage, on peut aisément parler de difficultés lors du passage de ce langage schwarz-bartien vers une 
autre langue. En anglais, le même mot " écume " n'a pas été rendu correctement non plus : " so much wind for such a little boat, so much mousse for so little chocolate ", que Kanhai-Burton juge mal traduit en anglais : " [it] is hardly relevant to the Caribbean context. Instead we can use the word 'froth' [...] since froth is what makes the cup of chocolate look like more than it is. While 'mousse' belongs to the world of affluent niceties, "froth' tells of the poverty of the Caribbean " (Kanhai-Burton, 1994, p. 50).

Ailleurs, des expressions créolisantes ont dû être sacrifiées pour des raisons de syntaxe : prenons l'imploration de Marie-Ange qui dénonce le sort des Haîtiens (du moins ceux de la " classe " à laquelle ils appartiennent, lui et elle) qui, nulle part, n'arrivent à se sentir chez eux, et à se faire une vie digne :

Wilnor, Wilnor, y a-t-il un pays où nous Haîti, on peut travailler, envoyer quelque argent chez soi, de temps en temps, sans se transfomer en courant d'air? (Schwarz-Bart, 1987, p. 17)

" Nous Haïti », sans être un créolisme, est ce que Tymoczko appelle « a marked feature of post-colonial writing »: une perturbation d'ordre lexical ou syntaxique indicatrice de la culture-source (Tymoczko, 1999, p. 24). Alors que Marie-Ange s'identifie en un même mouvement à son pays et à son peuple, une traduction mot à mot, rarement la meilleure solution, eût sauvé l'« étrangeté » du texte-source véhiculant d'autres normes, d'autres visions identitaires. Le côté évanescent de la langue, directement lié à l'intention postcoloniale, rend la traduction de Ton beau capitaine particulièrement épineuse. De fait, Schwarz-Bart résiste à toute interprétation réductrice en introduisant des trous, qui demandent à être remplis par le lecteur/spectateur créatif et qui rappellent la technique de la non-spécificité de Damas (Brown 1992). Contrairement au préjugé selon lequel une production tiers-mondiste serait transparente à la critique eurocentriste, Ton beau capitaine n'explique pas tout: l'Autre ne se laisse pas com-prendre facilement, comme Glissant épelait à juste titre le verbe. C'est que, trop longtemps, l'ex-colonisée a été regardé(e) avec condescendance : Schwarz-Bart inverse la situation. Elle met maintenant l'ex-colonisateur dans la situation embarrassée de ne pas tout comprendre, au point que certains critiques ont regretté le manque de clarté et d'intrigue (Silenieks, 1994, p. 522). Or, comme dans le nô, le public passe d'un état d'ignorance à une illumination plus ou moins grande. Ainsi, Wilnor brûle l'argent gagné en Guadeloupe, détail que les lecteurs/spectateurs s'expliquent 
mal, geste incontrôlé par lequel l'ex-colonisé accuse la fausseté et la trahison du matérialisme et du capitalisme.

\section{La Migration des caurs, Bovenwindse Hoogten}

Dans son introduction à la traduction anglaise des Derniers rois mages (Condé, 1992), Robert Philcox, traducteur et mari de Maryse Condé, attire l'attention sur l'acte cannibale qu'est la traduction et sur une dimension fondamentale de l'écriture condéenne qu'est l'ironie. L'introduction à The Last of African Kings a pour but de signaler à l'attention des lecteurs américains (et anglophones) l'intention moqueuse et l'irrévérence qui ne sont pas toujours aisées à déceler dans l'œuvre condéenne (Philcox 1997, IX), ni à traduire. Moi, ... Tituba, sorcière noire de Salem (1987) et Les Derniers rois mages sont particulièrement pétris de parodie (Scarboro, 1993). Considérant Condé avant tout comme une romancière imbue de vérité romanesque et de fidélité à l'Histoire, les Américains n'ont pas du tout perçu ses romans comme ironiques.

Quant à La Migration des coeurs, moins parodique il est vrai, la traduction nous défie une fois de plus. Commençons par le titre choisi par l'éditeur Franc Knipsheer: Bovenwindse Hoogten (qui donnerait littéralement Les Hauteurs du vent). Il désigne de manière plus explicite la réécriture condéenne de Wuthering Heights d'Emily Brontë. Or, l'épithète "Bovenwindse" renvoie à la distinction géographique caribéenne qui s'applique aux Antilles néerlandaises, et point aux Antilles françaises, la Martinique et la Guadeloupe étant désignées comme "Îles sous le vent" ("Benedenwindse Antillen »). Si le titre de la traduction affirme tout de suite le pastiche du roman brontéen, il me semble qu'en même temps, il s'éloigne trop de l'original, La Migration des caurs renvoyant à l'inconstance amoureuse, à la volubilité des cœurs. Face à ce registre émotionnel, "Bovenwindse Hoogten " renvoie à l'opposition, dans l'archipel caraïbe, entre Bovenwindse et Benedenwindse eilanden, si bien que le titre localise tout de suite l'histoire romanesque, alors qu'elle se déroule à Cuba, à la Dominique et à la Guadeloupe (les deux dernières étant des " Îles sous le Vent »).

Un autre point qui mérite notre attention est l'emploi des mots créoles et étrangers, non français, dans le corpus romanesque. Auteure qui refuse d'être étiquetée, Condé s'est montrée assez versatile, changeant d'un roman à l'autre l'emploi et le dosage de termes créoles 
et étrangers (Mesh-Ferguson 1994). Alors que Migration est dépourvu de notes et de glossaire, la traduction comporte des notes en fin de volume. Jetons-y un coup d'œil. La première remarque serait son caractère arbitraire. Un mot comme santería reçoit comme explication: "Cubaanse natuurgodsdienst" (religion animiste cubaine), ce qui donne, par substitution dans la traduction, une redondance $:$ p. 13 santeria-godsdienst. De plus, pareille définition n'explique pas l'origine africaine du culte syncrétique. Il eût mieux valu faire référence au vaudou en Haïti (avec les " loa's ") et à la macumba au Brésil. Ailleurs, certains termes s'accompagnent d'une note explicative dans le glossaire, mais il est fâcheux qu'ils soient restés sans astérisque dans le texte : bakwa's (transcription du créole « bakoua ») (p. 59).

Le plus regrettable est que la traductrice se soit référée au contexte surinamien qui n'est en rien identique à celui des Antilles françaises. Chaque île caribéenne a, en dépit de points communs, ses spécificités (qu'elles soient culinaires, botaniques, etc.). Traduire la couleur locale de la Guadeloupe et des petites Antilles par des référents surinamiens donne des termes inacceptables, tels que " mengwaterhindoestaan » dont le premier élément " mengwater » renvoie certes au métissage, mais le second aux Hindous, communauté démographiquement bien plus importante qu'aux Antilles. Par ailleurs, les descendants hindous(tans) y sont appelés "coolies", et ne représentent que $9 \%$ de la population. Ainsi, un terme à ma connaissance étranger aux Antilles françaises, tjemara (p. 38), est un autre emprunt à la botanique surinamienne, équivalent de «filao » : échanger un terme botanique local contre un autre ne résout en rien les problèmes de compréhension, et même si l'on peut espérer ou supposer que pas mal de lecteurs aux Pays-Bas connaissent la flore et la faune, la cuisine et la culture de leurs colonies, un lecteur belge bute contre le sème étranger, cette fois-ci laissé sans glose.

Ailleurs, Razyé, le Heathcliff noir, s'assoit dans un bar havanais pour boire " un mojito », traduit par « sopi » alors qu'il s'agit d'un apéritif à base de rhum avec une feuille de menthe, du sirop de canne, de la glace pilée, boisson cubaine typique. D'autres exemples de traduction impropre sont "Créole de sang mêlé » (Migration, p. 16), terme par lequel Condé désigne un "Blanc né aux îles », mais qui a du sang noir dans les veines, donc un métis, ce que l'on traduit une fois de plus par le terme surinamien : " mengbloed-creool », alors que le terme "Créole », appliqué à des individus, a de toutes autres significations aux Antilles qu'au Surinam. Traduire la littérature guadeloupéenne tout 
en restant fidèle à l'original demeure un défi. Puisqu'il s'agit ici d'une traduction interculturelle et que ce domaine reçoit enfin une attention critique croissante, l'on peut espérer que philologues et critiques littéraires travaillent main dans la main. Ceci au bénéfice des traductions, et donc des lecteurs qui tous, on ose l'espérer, en sortiront persuadés de l'importance d'une meilleure compréhension entre les cultures et que toute traduction équivaut à une re-contextualisation (Ming 1996, pp. 86-87).

Rendre la créolité de Ton beau capitaine et de La Migration des caurs n'est point une sinécure. Au même titre que l'auteur(e) créolophone tend des fils à un public francophone, le traducteur se trouve devant le défi de rester fidèle au "métissage " des langues et devrait plaider pour la différenciation, la différe/ance, respectant l'hétérogénéité. Comme pour Mariotte, la narratrice d'Un plat de porc aux bananes vertes (Simone et André Schwarz-Bart, 1967), la présence de plusieurs langues rend ce travail épineux, menaçant toujours que l'une ou l'autre langue soit reléguée à l'arrière-plan. Appendice exotisant de la littérature métropolitaine, les romans antillais requièrent une traduction précautionneuse, respectant la créolité du texte-source.

Université d'Anvers

\section{Références}

ALVAREZ-BORLAND, Isabel (1998). Cuban-American Literature of Exile. From Person to Persona. UP Virginia.

ARION, Frank Martinus (1998). Dubbelspel [1972]. Double Play. Trad. Paul Vincent. London, Faber and Faber.

ARTEAGA Alfred, ed. (1994). An Other Tongue. Nation and Ethnicity in the Linguistic Borderlands. Duke UP.

BALUTANSKY, Kathleen and Marie-Agnès SOURIEAU, eds. (1998). Caribbean Creolization. Reflections on the Cultural Dynamics of Language, Literature, and Identity. UP Florida/UP West Indies.

BASSNETT, Susan and Harish TRIVEDI, EDS. (1999). Post-Colonial Translation. Theory and Practice. London, Routledge.

BEKRI, Tahar (1999). « Écrire en deux langues ou le principe de vases 
communicants ». De la littérature tunisienne et maghrébine (et autres textes). Paris, L'Harmattan, pp. 109-119.

BENITEZ-ROJO, Antonio (1992). The Repeating Island. The Caribbean and the Postmodern Perspective. Trad. James E. Maraniss. Durham/London, Duke UP.

BERNABÉ, Jean (1979). "Contribution à l'étude de la diglossie littéraire : le cas de Pluie et Vent sur Télumée Miracle ". Textes, Études et Documents, 2, pp. 103-130.

BERNABÉ, Jean, Patrick CHAMOISEAU et Raphaël CONFIANT (1993). Éloge de la créolité/ In Praise of Creoleness. Trad. M.B. TalebKhyar. Paris, Gallimard.

BONA, Dominique (1998). Le manuscrit de Port-Ébène. Paris, Grasset.

BOYCE-DAVIES, Carole, ed. (1995). Moving Between Boundaries, Black Women's Diasporas, Critical Responses and Conversations, vol. 2. London, Pluto Press.

BROEK, Aart G. (1990). The Rise of the Caribbean Island's Literature. The Case of Curaçao and Its Writing in Papiamentu, Ph.D. Thesis. University of Amsterdam.

BROWN, Thomas H. (1992). "Filling in Reader Gaps in Poems by Léon-Gontran Damas ». French Literature Series, $\mathrm{n}^{\circ}$ 19, pp. 47-56.

CAIRO, Edgar (1993). Mindworry. A Surinamese Story of Father and Son (Temekoe, Kopzorg). Amsterdam, In de Knipscheer Publishers.

CALLALOO (1998). Caribbean Literature of Suriname, The Netherlands Antilles, Aruba and the Netherlands, A special issue, 21.3.

CÉSAIRE, Aimé (1983). Cahier d'un retour au pays natal (1939). Paris, Présence Africaine.

CHAMOISEAU, Patrick et Raphaël CONFIANT (1991). Lettres créoles, Tracées antillaises et continentales de la littérature 16351975. Paris, Hatier. 
CONDÉ, Maryse (1976). La poésie antillaise. Paris, Nathan.

- (1977). Le roman antillais. Paris, Nathan.

- (1986). Moi, Tituba... sorcière noire de Salem. Paris, Mercure de France. (Ik, Tituba...Zwarte heks van Salem, Amsterdam, In de Knipscheer Publishers, 1989).

- (1989). Traversée de la mangrove. Paris, Mercure de France. (Tocht door de mangrove, Amsterdam, In de Knipscheer Publishers, 1991).

- (1992). Les derniers rois mages. Paris. (Laatste Koningszoon, Amsterdam, In de Knipscheer Publishers, 1995).

- (1993). La Colonie du nouveau monde. Paris, Mercure de France. (Kolonie van de Nieuwe Wereld, Amsterdam, In de Knipscheer Publishers, 1997).

- (1995). La Migration des caurs. Paris, Laffont.

- (1997). Pays mêlé, nouvelles. Paris, Laffont.

- (1998). The Last of the African Kings. Trad. Richard Philcox. London, Nebraska University Press.

DAMAS, Léon-Gontran (1972). Pigments. Névralgies. Paris, Présence africaine.

DASH, Jean-Michael (1998). The Other America. The Caribbean in a New World Perspective. Charlottesville, Virginia University Press.

DEBROT, Cola (1958). My Sister the Negro. Trad. Estelle ReedDebrot. Pages from a Diary in Geneva. Amsterdam, Bezige Bij.

DEGRAS, Priska (1995). L'Héritage de Caliban. Éd. Maryse Condé. Paris, Karthala, pp. 85-101.

FANON, Frantz (1952). Peau noire, masques blancs. Paris, Seuil, coll. «Points».

GAUVIN, Lise (1997). L'écrivain à la croisée des langues. Paris, Karthala. 
- (2000). Langagement. L'écrivain et la langue au Québec. Montréal, Boréal.

GLISSANT, Édouard (1981). Le discours antillais. Paris, Gallimard.

- (1997). Traité du Tout-monde. Poétique IV. Paris, Gallimard.

GYSSELS, Kathleen (1997). «Frank Martinus Arion et Aimé Césaire, l'Afrique dans l'imaginaire afro-caribéen ". Septentrion, Arts et culture de Flandre et des Pays-Bas, 4, pp. 9-16.

HAMBUCH, Doris (forthcoming). "Walcott vs Naipaul : Intertextuality in Frank Martinus Arion's De laatste vrijheid ». Journal of Caribbean Literatures; 2.5 .

HARRIS, Jessica and Catherine TEMERSON (1989). "Your Handsome Captain 》. Callaloo, 12 :1, pp. 531-543.

HELMAN, Albert (1993). Chieftains of the Oayapok! (Hoofden van de Oayapok!). Trad. Scott Rollins. Amsterdam, In de Knipscheer Publishers.

HERNANDEZ, Elizabeth \& Consuelo Lopez SPRINGFIELD (1994). "Women and Writing in Puerto-Rico: An Interview with Ana Lydia Vega ». Callaloo, $17: 3$, pp. 816-825.

KADISH, Doris Y. and Françoise MASSARDIER-KENNEY (1996). "Traduire Maryse Condé, entretien avec Richard Philcox". The French Review, 69, April, pp. 749-761.

KADISH, Doris Y. (1997). «Moi, Tituba... sorcière noire de Salem et sa traduction ", L'ouvre de Maryse Condé. À propos d'une écrivaine politiquement incorrecte. Actes du colloque (Guad 14-18 mars 1995). Paris, L'Harmattan, pp. 231-247.

KANHAI-BURTON, Rosai (1994). « How Handsome can the Captain be? Translation of Simone Schwarz-Bart's Ton Beau capitaine within the Postcolonial Text ». Translation Review, 46, pp. 46-51.

LANG, George. (1997). « Islands, Enclaves, Continua: Notes Toward a Comparative History of Caribbean Creole Literatures ». A History of 
Literature of the Caribbean, Vol. 3. Ed. James A Arnold. Amsterdam/Philadelphia, John Benjamins, pp. 29-56.

- (2000). Entwisted Tongues. Comparative Creole Literatures. Amsterdam/Atlanta, Rodopi.

MAGNIER, Bernard (2000). «J'écris comme je vis ». Entretien avec Dany Laferrière. Gémenos, la Passe du Vent.

MESH-FERGUSON, Cynthia (1994). Language Conflict and the Francophone Guadeloupean Novel. An Interdisciplinary Inquiry, Ph.D. Thesis. Yale University Press.

MING, Xie (1996). «Intercultural Translation, Distance and Appropriation ». Perspectives in Translatology, Vol. 471, pp. 71-89.

MOURA, Jean-Marc (1999). Littératures francophones et théorie postcoloniale. Paris, Presses universitaires de France.

ONWUNEMENE, Michael C. (1999). " Limits of Transculturation: Nigerian Writer's Endeavors toward a National Literary Language ». $P M L A, 114$, October, pp. 1055-1066.

PFAFF, Françoise (1993). Entretiens avec Maryse Condé. Paris, Karthala.

POS, Hugo (1994). Moving Stories. Amsterdam, In de Knipscheer Publishers.

ROEMER, Astrid (1994). A Name for Love (Een naam voor de liefde). Trans. Rita Gircour. Amsterdam, In de Knipscheer Publishers.

- (1994). The Order of the Day (De orde van de dag) (1988, Conserve). Trad. Rita Gircour. Amsterdam, In de Knipscheer Publishers.

ROWELL, Charles H (1998). « Interview with Frank Martinus Arion ». Callaloo, 21:3, pp. 538-541.

RUTGERS, Wim (1994). Schrijven is zilver, Spreken is goud, Auratuur, Oratuur en Literatuur van de Nederlandse Antillen en Aruba, Ph.D. Thesis. Universiteit Utrecht. 
SCARBORO, Ann Armstrong (1993). « Afterword » in I, Tituba, Black Witch of Salem. Trad. Robert Philcox. University of Virginia, pp. 187225, " Caraf Books ».

SCHWARZ-BART, André et Simone (1967). Un plat de porc aux bananes vertes. Paris, Seuil. (Een schotel varkensvlees met groene banaan, Amsterdam, In de Knipscheer Publishers, trad. C.P. HeeringNoorman, 1993).

SCHWARZ-BART, Simone (1972). Pluie et vent sur Télumée Miracle. Paris, Seuil, Coll. " Points». (Wind en Zeil, Amsterdam, In de Knipscheer Publishers, trad. Edith Klapwijk, 1986).

- (1979). Ti Jean L'horizon. Paris, Seuil, coll. "Points ». (Horizont, Amsterdam, In de Knipscheer Publishers, trad. Hetty Renes, 1983).

- (1987). Ton beau capitaine. Paris, Seuil. (Je knappe kapitein, Amsterdam, In de Knipscheer Publishers, trad. Eveline van Hemert, 1993).

SILENIEKS, Juris (1994). " Marronnage and the Canon. Theater to the Negritude Era ". A History of Literature of the Caribbean. Hispanic and Francophone regions, Vol. 1. Ed. James A. Arnold. Amsterdam/Philadelphia, John Benjamins, pp. 507-525.

TALEB-KHYAR, B (1991). « An Interview with Maryse Condé and Rita Dove ». Callaloo, 14 :2, pp. 347-66.

TODOROV, Tzvetan (1994). "Dialogism and Schizophrenia ». An Other Tongue. Nation and Ethnicity in the Linguistic Borderlands. Ed. Alfred ARTEAGA. Durham/London, Duke University Press, pp. 203214.

TYMOCZKO, Maria (1999). «Post-colonial Writing and Literary Translation ". Post-Colonial Translation, Theory and Practice. Eds. Susan BASSNETT and Harish TRIVEDI. London and New York, Routledge, pp. 19-40.

VAN KEMPEN, Michiel (1989): Surinaamse schrijvers en dichters. Amsterdam, Arbeiderspers. 
VAN LEEUWEN, Boeli (1993). Shields of Clay (Schilden van leem). Amsterdam, In de Knipscheer Publishers.

- (1993). The Sign of Jonah. Amsterdam, In de Knipscheer Publishers.

VECIANA-SUAREZ, Ana (1999). The Chin Kiss King. Newark, Pinguin Putman.

VENUTI, Lawrence (1998). The Scandals of Translation. Towards an Ethics of Difference. London, Routledge.

VIZENOR, Gerald (1994). "The Ruins of Representation »: An Other Tongue, Nation and Ethnicity in the Linguistic Borderlands. Ed. Alfred Arteaga. Durham and London, Duke University Press, pp. 138-167.

VERSTRAETE, Benoît, éd (1997). L'émancipation dans la littérature néerlandophone des Caraïbes/Emancipatie in de Nederlandstalige Caraïbische literatuur. Lille, Lille III/La Maison Coornhert.

WALCOTT, Derek (1992). The Star Apple Kingdom. Royaume du fruit-étoile. Strasbourg, Éd. Circé.

RÉSUMÉ : La migration des mots et le néerlandais comme « langue mineure » dans la mosaïque linguistique caribéenne Parmi les diverses littératures antillaises, la littérature des îles néerlandaises est méconnue et peu étudiée. Cet article analysera d'abord les raisons de cette paucité et soulignera en même temps quelques rares initiatives visant à sortir de cet isolement linguistique : des numéros spéciaux de revues littéraires ont été dédiés à la promotion et à l'émancipation des auteurs caribéens-néerlandais. Dans un deuxième temps, l'article met l'emphase sur l'importance de la traduction en hollandais de la littérature antillaise francophone. L'éditeur hollandais Knipscheer a privilégié les auteurs caribéens francophones et nous nous penchons dans une dernière partie sur quelques-unes des difficultés que pose la traduction en hollandais des romans de Simone Schwarz-Bart et de Condé.

ABSTRACT: Word Migration and Dutch as a "Minor" Caribbean Language - Among the various Caribbean literatures, DutchCaribbean literature remains largely unknown and unstudied. In a first 
part, this article analyzes the reasons for this paucity, and indicates at the same time some rare initiatives to break out of the linguistic isolation: special issues of outstanding literary reviews have been dedicated to the promotion and emancipation of Dutch Caribbean writers. In a second part, the article stresses the importance of translation of Francophone Caribbean literature into Dutch: the Dutch editor Knipscheer has given extensive attention to French Caribbean authors, and we focus in a last part on some difficulties in translating Simone Schwarz-Bart's and Condé's novels into Dutch.

Mots-clés : traduction interculturelle, créolisation et créolité, littérature néerlandophone caribéenne, littérature francophone caribéenne en néerlandais, interlangue.

Key words: intercultural translation, creolisation and "caribbeanness", Dutch Caribbean literature, French Caribbean literature in Dutch, "interlangue".

Kathleen Gyssels : UFSIA, Prinsstraat 13, B-2000 Antwerpen

Courriel : Kathleen.gyssels@ua.ac.be 